\title{
BMJ Open Experience of living with cancer and comorbid illness: protocol for a qualitative systematic review
}

\author{
Debbie Cavers, ${ }^{1}$ Sarah Cunningham-Burley, ${ }^{1}$ Eila Watson, ${ }^{2}$ Elspeth Banks, ${ }^{3}$ \\ Christine Campbell ${ }^{1}$
}

To cite: Cavers D,

Cunningham-Burley $\mathrm{S}$,

Watson $\mathrm{E}$, et al. Experience of living with cancer and comorbid illness: protocol for a qualitative systematic review. BMJ Open 2017;7:e013383. doi:10.1136/ bmjopen-2016-013383

- Prepublication history and additional material is available. To view please visit the journal (http://dx.doi.org/10.1136/ bmjopen-2016-013383)

Received 12 July 2016 Revised 7 December 2016 Accepted 16 December 2016

CrossMark

${ }^{1}$ The Usher Institute of Population Health Sciences and Informatics, Medical

School, University of Edinburgh, Edinburgh, UK

${ }^{2}$ Faculty of Health and Life Sciences, Oxford Brookes University, Oxford, UK ${ }^{3} \mathrm{NCRI}$ Psychosocial Oncology and Survivorship Clinical Studies Group, London, UK

Correspondence to

Dr Debbie Cavers;

debbie.cavers@ed.ac.uk

\section{ABSTRACT}

Introduction There are an increasing number of people living with and beyond cancer, whose experience is further complicated by additional long-term health conditions in the context of an ageing population. The supportive care needs of this growing patient group should be recognised and addressed. There is a need to explore the experience of living with cancer and comorbid illness in order to develop optimal models of patient-centred care. This protocol describes a systematic review that aims to identify the qualitative evidence relating to the experience of cancer and comorbid illness for patients, informal carers and professionals, and to highlight areas where more research is needed.

Methods and analysis A systematic review following PRISMA guidance will be undertaken. Medline, Embase, CINAHL, PsycINFO, ASSIA, Sociological Abstracts, Web of Science, SCOPUS, OpenGrey and ProQuest Dissertations and Theses Global databases will be systematically searched for articles relevant to patient, carer and professional experiences. Two independent reviewers will screen articles for inclusion and evaluate them according to the Critical Appraisal Skills Programme tool. Extracted data will be combined using recognised methods of qualitative synthesis to offer new insights into the topic area and for a patient-centred model of care.

Ethics and dissemination The review does not require formal ethical review as no direct patient contact or patient identifiable data is used. Conduct of the review has been approved internally by the University of Edinburgh Centre for Population Health Sciences Ethics Review Committee. Results of the review will be published in a generalist peerreviewed journal and presented at a relevant conference in addition to informing subsequent empirical work by the authors on this topic area.

\section{INTRODUCTION}

Due to the ageing population and better screening, diagnosis and treatments, there are an increasing number of people living with and beyond cancer. ${ }^{1}$ The lived experience of these individuals is often complicated by other comorbid chronic illnesses; as many as $78 \%$ of people with cancer are living with at least one other condition, the prevalence of which also increases with age. ${ }^{2} 3$ Therefore, the support needs of people living with
Strengths and limitations of this study

Protocol for a systematic review of current literature exploring a highly important topic presenting a current challenge to healthcare provision

- Using robust methodology following standardised guidelines

- Scope to inform policy and practice and improve patient care

- Systematic review of qualitative empirical evidence only; does not cover quantitative studies or existing systematic reviews but can give important insights

- Large evidence base for experience of cancer and experience of multimorbidity, but little linking the two bodies of evidence, presenting a challenge to the reviewers

multiple complex conditions are enduring and need to be identified and addressed. ${ }^{4}$ In addition to the challenges facing secondary care ${ }^{5}$ there is increasing recognition of the role of primary care in the provision of ongoing support to patients with cancer, particularly after the completion of hospital-based treatment as patients adjust to life post-treatment, living with ongoing medical issues related to their cancer treatment. ${ }^{67}$ This picture becomes more complex in the presence of other long-term conditions, with implications for the coordination of quality care and support. ${ }^{89}$

Meeting the needs of people living beyond cancer has been identified as a policy priority, ${ }^{10}$ which informed the development of the National Cancer Survivorship Initiative $^{6}$ in England and Wales; survivorship also forms part of the remit for Scotland's Better Cancer Care. ${ }^{11}$ In many cases, survivorship initiatives and research target the clinical needs related to long-term side effects. ${ }^{12}$ However, psychosocial support for survivors of cancer is another important consideration and has implications for the role of primary, secondary and community care. While valuable research has been conducted to 
understand better these dimensions of life for the patient and their relatives ${ }^{13-17}$ less is known about the impact of additional chronic illness on patients' lived experience. As such, service development and provision would benefit from further in-depth research in this area. ${ }^{71}$ Understanding the challenges experienced by people living after a cancer diagnosis with other chronic conditions such as chronic obstructive pulmonary disease, diabetes or mental ill health can give new insights into patient-centred models of care.

\section{Objectives}

While addressing the holistic needs of cancer survivors forms part of the vision of survivorship initiatives, and there is a growing body of work exploring experience of multimorbidity, ${ }^{19}$ there is a dearth of research linking cancer with comorbid disease to further enrich our understanding of living with such complex illness. This systematic review aims to synthesise and report on published evidence exploring the experience of cancer survivorship in connection with that of other long-term conditions in order to identify critical research questions. The findings from the review will combine with those from a separate patient-centred research prioritisation exercise to set a qualitative empirical research agenda for future work.

\section{Research questions}

- What are the findings of qualitative evidence exploring the experience of living with both cancer and one or more comorbidities from patient, carer and provider perspectives?

- What are the psychosocial support needs of people living with cancer and one or more other long-term condition(s) identified in the literature?

- What are patient, carer and provider experiences of service provision reported in the literature?

- What research priorities can be derived from the available evidence?

\section{REVIEW METHODS}

\section{Design}

A systematic review of the current evidence linking experience of cancer with comorbid illness is considered a robust way of identifying and synthesising the published evidence in this area to define a cohesive empirical research agenda that builds on prior knowledge. ${ }^{20}$ The review will include qualitative evidence only to produce an interpretation built on people's views and experiences, acknowledging the rich context and different dimensions of the lived experience from the perspective of those experiencing it. Further, a synthesis of qualitative data aims to generate findings that are meaningful, relevant and appropriate to individuals, to inform a qualitative research agenda and ultimately to more effectively influence policy and practice influencing patient outcomes. ${ }^{21}$

The review is based on the PRISMA statement guidance for conducting a systematic review, ${ }^{22}$ and the protocol follows the relevant items on the online supplementary PRISMA-P checklist). ${ }^{23}$ The review protocol has been registered on the International Prospective Register of Systematic Reviews (PROSPERO) Database (registration number: CRD42016041796).

The review will use methods of qualitative synthesis to combine, integrate and interpret, where possible, the evidence from the included papers (see Eligibility criteria and Data synthesis sections). ${ }^{21}$ The review aims to move beyond the aggregation of available data to provide further interpretive insights into living with complex illness and define where future research can add to what is known. ${ }^{24}$

No NHS ethical approval is required for this study as it will not include patient identifiable data. It has been internally approved by the University of Edinburgh Centre for Population Health Sciences Ethics Review Committee (Ref: 8/03/2016).

\section{Eligibility criteria}

The review will include qualitative empirical studies, including unpublished grey literature. Qualitative data from mixed methods studies will be screened for inclusion and included if the qualitative component is relevant. Included articles will be published between 2000 and the present day to ensure the currency of the work while enabling a broad view of developing issues to be identified. Articles will be included that address the lived experience of the cancer journey with attention to existing long-term health conditions (developed before or after the cancer diagnosis) and will identify issues related to psychosocial and supportive care as outlined in relation to the anticipated dimensions of interest. Articles will be included from across the cancer continuum from diagnosis through to end of life and will include the perspectives of adult patients (aged $\geq 18$ years), informal carers and healthcare professionals. The review will include only articles published in English.

Studies will be included for any cancer type in combination with one or more comorbid long-term condition (LTC), also described as cancer multimorbidity. Longterm conditions to be included will be guided by those listed in Barnett et al's paper mapping the epidemiology of multimorbidity ${ }^{3}$ (see online supplementary appendix 1 for a full list of conditions). The applied definition of long-term conditions is based on ISD Scotland's report on important long-term conditions in Scotland; the report uses, among others, the Long-Term Conditions Collaborative to define an LTC as, "one that requires ongoing care, limits what one can do and is likely to last longer than a year ${ }^{25}$ Long-term side effects of cancer treatment and second primary cancers are not included; experience of second primary cancers is being addressed separately within the research centre. Where it is not possible to determine whether the condition is caused by cancer treatment, the article will be included in the review but analysed separately. 


\section{Dimensions of interest}

The review focuses on an exploration of the dimensions of lived experience: physical, social, emotional and psychological. From a similar study exploring multiple dimensions of experience, these are expected to include such topics as quality of life, well-being, psychosocial supportive care needs, attitude and identity, perceived continuity and quality of care and influence of personal demographics (age, gender, ethnicity, socioeconomic status). ${ }^{26}$ In relation to how services can best meet patients' needs, topics may include issues of accessing appropriate services, coordination of care for multiple conditions and self-management across the cancer care continuum. ${ }^{1719}$ The search strategy has been developed to reflect these broad and exploratory domains. The outcomes will depend on the evidence available, and gaps in the evidence will be highlighted for future study.

\section{Information sources}

A variety of search strategies will be used to identify potential literature for the review, particularly given the challenges in identifying qualitative evidence. ${ }^{27}$ This will primarily involve a literature database search (including grey literature), but will also include citation and snowball searching, known expert consultation via email, related articles searches in PubMed and use of Google scholar. The databases to be consulted are: Medline, Embase, CINAHL, PsycINFO, ASSIA, Sociological Abstracts, Web of Science, SCOPUS and, for grey literature, OpenGrey and ProQuest Dissertations and Theses Global. These are considered sufficient to comprehensively cover the range of topics and disciplines implicated in this review.

\section{Search strategy}

The searching phase aims to identify relevant studies for inclusion in the review. The process of searching will begin with individually searching each of the listed databases using an adapted list of search terms; the developed search strategy for Medline is shown in box 1 . The search terms will be adapted to suit each database to derive the most meaningful search and will use free text, MeSH and subject headings for maximum sensitivity and specificity.

\section{DATA COLLECTION AND ANALYSIS \\ Study records}

Identified records will be imported into and managed in EndNoteX7. Screened and selected articles will be managed in subsequent EndNote databases in order to track and record the number of records retained at each step. It is anticipated that, given the relative lack of attention in the literature specifically examining the experience of long-term conditions as a cancer survivor, the review will produce a small number of relevant but heterogeneous studies.

\section{Screening}

Screening of articles will adhere to the specified inclusion criteria (as detailed earlier; also see box 2).
A three-step screening process will be undertaken to achieve the final set of included articles. First, all titles will be read and those of no obvious relevance will be excluded. This process will be undertaken by the primary reviewer DC. Second, abstract screening will be undertaken by DC and another reviewer to look in more detail for relevance and fit with the inclusion criteria. Any differences in judgement will be resolved by a third reviewer. Finally, full-text articles of remaining studies will be obtained and read, again by DC and one other reviewer, to assess their suitability for inclusion in the final review. Full-text articles deemed to meet the inclusion criteria will be selected and subject to quality appraisal and data extraction.

\section{Data extraction}

Data extraction will be managed in Microsoft Excel using a purpose designed proforma. Extracted material will reflect the inclusion criteria and the designated aims of the review, derived from the article as a whole. Information will be gathered on: author; year of publication; country of study; study type; setting; relevant background and impetus for the study; methodological approach and specified methods; patient characteristics and demographics including cancer and comorbidity type; main findings including pertinent themes relating to experience of illness, psychosocial needs and supportive care; strengths and limitations and key relevant discussion points. Extraction of data will be carried out by DC and the second reviewer.

\section{Quality assessment}

All included articles will be subject to critical appraisal using the Critical Appraisal Skills Programme (CASP) tool. The 10-item CASP tool was considered to be the most suitable tool to consider the quality parameters of qualitative work and is a well-validated and accepted tool. In reference to the debate on exclusion of qualitative articles on the basis of quality, no articles will be excluded on these grounds alone and a low threshold for inclusion will be applied so that the review can benefit from researcher insight and theoretical as well as empirical contributions. ${ }^{28-30}$ The relative quality of included studies will be critically considered during the analysis and in reference to the developed synthesis. Quality assessment will be undertaken by two independent reviewers, and any differences of opinion will be discussed and reviewed by a third person if no consensus is reached.

\section{Data synthesis}

For a qualitative review seeking to explore personal lived experiences, it was important to find a method that acknowledges the different paradigms and positions on which each research output is based as well as allowing for different study methods covering a broad and exploratory topic area. ${ }^{31}$ Meta-ethnography, used originally in education research by Noblit and Hare but has more recently been of interest in nursing and health services research,,$^{32-34}$ was considered as an appropriate method 


\section{Box 1 Example search strategy for Medline}

1. Comorbidity/

2. (Multimorbid\$ or multi-morbid\$ or Co-morbid\$).mp. [mp=title, abstract, original title, name of substance word, subject heading word, keyword heading word, protocol supplementary concept word, rare disease supplementary concept word, unique identifier]

3. Chronic Disease/ or (Chronic adj5 (illness $\$$ or condition\$ or disease)).tw.

4. (Long term adj5 (condition\$ or illness\$ or disease\$)).mp. [mp=title, abstract, original title, name of substance word, subject heading word, keyword heading word, protocol supplementary concept word, rare disease supplementary concept word, unique identifier]

5. 1 or 2 or 3 or 4

6. Neoplasms/ or Cancer.mp or (Tumour or tumor or Oncology or Neoplasm).mp. [mp=title, abstract, original title, name of substance word, subject heading word, keyword heading word, protocol supplementary concept word, rare disease supplementary concept word, unique identifier]

7. Qualitative Research/ or Qualitative.mp or Interview/ $r$ Interview\$.mp or In-depth.mp

8. (Focus Group or Ethnograph\$ or Observation\$ or Participant\$ or Respondent\$ or View\$ or Belief\$ or Attitude\$ or Awareness or Perspective\$ or Understanding\$ or Findings or Grounded Theory or Social Construction\$ or Theoretical or Phenomenolog\$).mp. [mp=title, abstract, original title, name of substance word, subject heading word, keyword heading word, protocol supplementary concept word, rare disease supplementary concept word, unique identifier]

9. 7 or 8

10. Psychosocial.mp. or Stress, Psychological/ or Social Support/ or Psycho-social.mp or 'Quality of Life'/ or 'Experience of IIIness'.mp or Experience adj5 IIIness or Attitude to Health/ or 'Quality of Life'.mp or Happ\$.mp or Emotion\$.mp. or Emotions/

11. Patient satisfaction.mp. or Patient Satisfaction/ or 'Quality of Health Care'/ or 'Patient Experience'.mp or Satisfaction.mp or 'Information Preferences'.mp or Decision Making/ or (Decision-making or Decision Making).mp or (Informed choice or Informed Decision Making).mp or Self management.mp. or Self Care/ or 'Continuity of Patient Care'/ or Integrated care.mp

12. Access to Health Care.mp. or Health Services Accessibility/

13. Identity.mp. or gender.mp. or Gender Identity/

14. (Health behaviour or Health behavior).mp.

15. (Help-seeking or Help seeking).mp.

16. (Well-being or Well being).mp. or Holistic.mp or Continuity of Care.mp or 'Continuity of Patient Care'/ or (Fragmented adj5 care).mp or (joined-up or Joined Up).mp or Integrated care.mp or Diversity of Care.mp.

17. Primary care.mp. or Primary Health Care/ or Secondary care.mp. or Secondary Care/ or Community Care.mp or Community Health Services/ or Ambulatory Care.mp. or Ambulatory Care/ or 'Delivery of Health Care, Integrated'/ or Preventive Health Services/ or Family Practice/ or Shared care.mp or Collaborat\$ care.mp

18. (Patient-centred care or Patient centred care or Patient-centred or Person-centred or Patient centred or Person centred).mp or PatientCentered Care/

19. Palliative Care/ or Supportive care.mp. or palliative.mp.

20. 'Health Services Needs and Demand'/ or unmet need.mp. or Health Services Research/ or Depriv\$.mp. or (Socio-economic or Socioeconomic).mp. or Information needs.mp.

21. Survivor\$.mp. or Survivors/

22. Patient Care Planning/ or Care plan.mp. or (Follow-up or Follow up).mp.

23. ('use of service \$' or 'service use' or 'service adj5 use').mp.

24. 10 or 11 or 12 or 13 or 14 or 15 or 16 or 17 or 18 or 19 or 20 or 21 or 22 or 23

25. 5 AND 6 AND 9 AND 24

\section{Box 2 Inclusion and exclusion criteria}

\section{Inclusion criteria}

- Study population -Adults (over 18) with a diagnosis of cancer and at least one other LTC (as specified in online supplementary appendix 1), carers and health professionals

- Articles relating to experience of illness from patient, carer and professional perspectives

- Articles focusing on areas denoted in the dimensions of interest

Studies with a qualitative empirical design

- Articles published in English

\section{Exclusion criteria}

Articles not meeting the above inclusion criteria

- Articles published before 2000

- Articles focusing on long-term side effects of cancer treatment or second primary cancers alone for this purpose as it is suited to reviewing a small body of exclusively qualitative evidence and gives rise to a meaningful interpretive account rather than simply aggregating the data. ${ }^{3235}$ However, thematic synthesis, developed by Thomas and Harden, was also considered as it provides a prescriptive approach that lends itself well to transparency and quality in the conduct and reporting of the review, and in generating hypotheses to meet the review's objective of generating further empirical research questions. $^{2124}$ Like meta-ethnography, this method encourages the researcher to consider the concepts and themes that relate different studies-through line by line coding, developing descriptive themes and generating analytic themes - to provide a mutual translation and ultimately produce an interpretive account of the phenomenon of 
interest, as a grounded theory approach aims to do with primary data analysis. ${ }^{37} 38$

Given the anticipated yield of a small heterogeneous body of evidence meeting the eligibility criteria for the review, a narrative-based summary may be more appropriate and achievable using steps one and two of Thomas and Harden's thematic synthesis. ${ }^{21}$ Extracted data will be combined and described using the most appropriate method to reflect the evidence available. Where possible, an interpretive account of the data will be presented in the context of a critical review of the evidence. The descriptive or conceptual outputs will be related to the research questions and objectives.

\section{DISCUSSION}

Insights from the proposed review will contribute to what is known, giving novel attention to the combination of cancer with other long-term chronic conditions. This is considered a necessary step in cancer survivorship research and care in the face of the increasing, ageing population and the shifting emphasis in patient care.

Building on the knowledge base will contribute to an evidence-based and conceptually informed integrated model of care, with an emphasis on better understanding experiences of complex and multiple conditions and the implications for living with cancer and comorbid illness in the push towards shared care or self-management. ${ }^{39}$

While conceptual and theoretical insights to a subject area can add richness to the academic evidence base, it is important to produce a robust qualitative synthesis that reflects the identified evidence. ${ }^{24}$ A narrative summary can adequately meet the proposed objectives, provide answers to the research questions and speak meaningfully to policy directives.

The findings from the review will combine with patient engagement work by the authors to inform an empirical study exploring the needs of the defined patient group and the development of an intervention to better address their complex needs. The review will be published in a peer-reviewed journal and presented at relevant conferences in addition to being shared with local interest groups and via online social media.

Acknowledgements We thank Ms Marshall Dozier, Academic Liaison Librarian, for advice on designing the review and searching literature databases and Dr Karen Barnett for advice on defining included long-term conditions.

Contributors DC designed the systematic review with input from CC, SC-B, EW and EB. DC drafted the review protocol with input from CC, SC-B, EW and EB.

Funding This review has been funded by the Scottish Government's Chief Scientist Office (CSO); funding reference number: PDF/15/06. The CSO have provided financial support as part of a personal fellowship but are not involved in other aspect of the study design, implementation or analysis.

Competing interests None declared.

Provenance and peer review Not commissioned; externally peer reviewed.

Open Access This is an Open Access article distributed in accordance with the Creative Commons Attribution Non Commercial (CC BY-NC 4.0) license, which permits others to distribute, remix, adapt, build upon this work non-commercially, and license their derivative works on different terms, provided the original work is properly cited and the use is non-commercial. See: http://creativecommons.org/licenses/by-nc/4.0/ (c) Article author(s) (or their employer(s) unless otherwise stated in the text of the article) 2017. All rights reserved. No commercial use is permitted unless otherwise expressly granted.

\section{REFERENCES}

1. Information Service Division. Cancer in Scotland, 2014.

2. McLean G, Gunn J, Wyke S, et al. The influence of socioeconomic deprivation on multimorbidity at different ages: a cross-sectional study. Br J Gen Pract 2014;64:e440-e447.

3. Barnett K, Mercer SW, Norbury M, et al. Epidemiology of multimorbidity and implications for health care, research, and medical education: a cross-sectional study. Lancet 2012;380:37-43.

4. Mason B, Nanton V, Epiphaniou E, et al. 'My body's falling apart'. Understanding the experiences of patients with advanced multimorbidity to improve care: serial interviews with patients and carers. BMJ Support Palliat Care 2014;6:1-6.

5. Smith SM, Allwright S, O'Dowd T. Does sharing care across the primary-specialty interface improve outcomes in chronic disease? A systematic review. Am J Manag Care 2008;14:213-24.

6. NHS Health Improvement and Macmillan Cancer support. National cancer survivorship initiative: vision 2010

7. Smith SM, Soubhi H, Fortin M, et al. Managing patients with multimorbidity: systematic review of interventions in primary care and community settings. BMJ 2012;345:e5205.

8. Snyder CF, Frick KD, Herbert RJ, et al. Comorbid condition care quality in cancer survivors: role of primary care and specialty providers and care coordination. J Cancer Surviv 2015;9:641-9.

9. Williams GR, Mackenzie A, Magnuson A, et al. Comorbidity in older adults with Cancer. J Geriatr Oncol 2016;7:249-57.

10. Department of Health. Cancer reform strategy, 2007.

11. Scottish Government. Better cancer care: an action plan, 2008.

12. Adams E, Hill E, Watson E. Fertility preservation in cancer survivors: a national survey of oncologists' current knowledge, practice and attitudes. Br J Cancer 2013;108:1602-15.

13. Harrison SE, Watson EK, Ward AM, et al. Primary health and supportive care needs of long-term cancer survivors: a questionnaire survey. J Clin Oncol 2011;29:2091-8.

14. Adams E, Boulton M, Rose PW, et al. A qualitative study exploring the experience of the partners of cancer survivors and their views on the role of primary care. Support Care Cancer 2012;20:2785-94.

15. Watson EK, Rose PW, Loftus R, et al. Cancer survivorship: the impact on primary care. Br J Gen Pract 2011;61:763-5.

16. Watson EK, O'Brien R, Campbell C, et al. Views of health professionals on the role of primary care in the follow-up of men with prostate cancer.. Fam Pract 2011;28:647-54.

17. O'Brien R, Wyke S, Guthrie B, et al. An 'endless struggle': a qualitative study of general practitioners' and practice nurses' experiences of managing multimorbidity in socio-economically deprived areas of Scotland. Chronic IIIn 2011;7:45-59.

18. France EF, Wyke S, Gunn JM, et al. Multimorbidity in primary care: a systematic review of prospective cohort studies. Br J Gen Pract 2012;62:297-307.

19. Coventry PA, Small N, Panagioti M, et al. Living with complexity; marshalling resources: a systematic review and qualitative metasynthesis of lived experience of mental and physical multimorbidity. BMC Fam Pract 2015;16:171.

20. Hemingway P, Brereton N. What is a systematic review? 2009. http:// www.medicine.ox.ac.uk/bandolier/painres/download/whatis/systreview.pdf (accessed 31 Mar 2014).

21. Thomas J, Harden A. Methods for the thematic synthesis of qualitative research in systematic reviews. BMC Med Res Methodol 2008;8:45.

22. Moher D, Liberati A, Tetzlaff J, et al. Preferred reporting items for systematic reviews and meta-analyses: the PRISMA statement. PLOS Med 2009;6:e1000097.

23. Moher D, Shamseer L, Clarke M, et al. Preferred reporting items for systematic review and meta-analysis protocols (PRISMA-P) 2015 statement. Syst Rev 2015;4:1

24. Barnett-Page E, Thomas J. Methods for the synthesis of qualitative research: a critical review. BMC Med Res Methodol 2009;9:59.

25. Information Services Division. Measuring long term conditions in Scotland. NHS National Services Scotland 2008.

26. Cavers D, Hacking B, Erridge SC, et al. Adjustment and support needs of glioma patients and their relatives: serial interviews. Psychooncology 2013;22:1299-305.

27. Shaw RL, Booth $A$, Sutton AJ, et al. Finding qualitative research: an evaluation of search strategies. BMC Med Res Methodol $2004 ; 4: 5$ 
28. Mays N, Pope C. Qualitative research in health care. Assessing quality in qualitative research. BMJ 2000;320:50-2.

29. Dixon-Woods M, Shaw RL, Agarwal S, et al. The problem of appraising qualitative research. Qual Saf Health Care 2004;13:223-5.

30. Petticrew M, Roberts H. Systematic reviews in the social sciences: a practical guide. Blackwell Publishing, Oxford, UK, 2006.

31. Dixon-Woods M, Fitzpatrick R. Qualitative research in systematic reviews. BMJ 2001;323:765-6.

32. Dixon-Woods M, Cavers D, Agarwal S, et al. Conducting a critical interpretive synthesis of the literature on access to healthcare by vulnerable groups. BMC Med Res Methodol 2006;6:35.

33. Lang $\mathrm{H}$, France $\mathrm{E}$, Williams $\mathrm{B}$, et al. The psychological experience of living with head and neck cancer: a systematic review and metasynthesis. Psychooncology 2013;22:2648-63.
34. Britten N, Campbell R, Pope C, et al. Using Meta ethnography to synthesise qualitative research: a worked example. J Health Serv Res Policy 2002;7:209-15.

35. Noblit G, Hare R. Meta-Ethnography: synthesising qualitative studies. SAGE Publications, London, UK, 1988.

36. Campbell R, Pound P, Morgan M , et al Health Technology Assessment. Evaluating meta-ethnography: systematic analysis and synthesis of qualitative research 2011;15:1-164.

37. Glaser B, Strauss A. The discovery of grounded theory: strategies for qualitative research. Aldine Transaction, New Brunswick, USA, 1967.

38. Charmaz K. Constructing grounded theory: a practical guide through qualitative analysis. 2nd ed. SAGE, London, UK, 2013.

39. Coffey L, Mooney O, Dunne S, et al. Cancer survivors' perspectives on adjustment-focused self-management interventions: a qualitative meta-synthesis. J Cancer Surviv 2016;10:1012-34. 